\title{
Geodesic neighborhoods in unitary orbits of self-adjoint operators of $\mathcal{K}+\mathbb{C}$
}

\author{
Tamara Bottazzi ${ }^{\mathrm{a}, \mathrm{b}}$, Alejandro Varela ${ }^{\mathrm{c}, \mathrm{d}, *}$ \\ a Universidad Nacional de Río Negro, Centro Interdisciplinario de Telecomunicaciones, Electrónica, \\ Computación y Ciencia Aplicada, Sede Andina, (8400) S.C. de Bariloche, Argentina \\ b Consejo Nacional de Investigaciones Científicas y Técnicas, (1425) Buenos Aires, Argentina \\ c Instituto Argentino de Matemática "Alberto P. Calderón", Saavedra 15 3er. piso, (C1083ACA) Buenos \\ Aires, Argentina \\ d Instituto de Ciencias, Universidad Nacional de Gral. Sarmiento, J. M. Gutierrez 1150, (B1613GSX) \\ Los Polvorines, Argentina
}

\section{A R T I C L E I N F O}

\section{Article history:}

Received 6 April 2019

Received in revised form 7 May 2021

Accepted 19 May 2021

Available online $\mathrm{xxxx}$

Communicated by V. Soucek

\section{$M S C$ :}

primary $22 \mathrm{~F} 30,53 \mathrm{C} 22$

secondary 22E $65,47 \mathrm{~B} 15,51 \mathrm{~F} 25$,

$58 \mathrm{~B} 20$

Keywords:

Unitary orbits

Geodesic curves

Minimality

Finsler metric

\begin{abstract}
A B S T R A C T
In the present paper, we study the unitary orbit of a compact Hermitian diagonal operator with spectral multiplicity one under the action of the unitary group $\mathcal{U}_{\mathcal{K}+\mathbb{C}}$ of the unitization of the compact operators $\mathcal{K}(\mathcal{H})+\mathbb{C}$, or equivalently, the quotient $\mathcal{U}_{\mathcal{K}+\mathbb{C}} / \mathcal{U}_{\mathcal{D}(\mathcal{K}+\mathbb{C})}$. We relate this and the action of different unitary subgroups to describe metric geodesics (using a natural distance) which join end points. As a consequence we obtain a local Hopf-Rinow theorem. We also explore cases about the uniqueness of short curves and prove that there exist some of these that cannot be parameterized using minimal anti-Hermitian operators of $\mathcal{K}(\mathcal{H})+\mathbb{C}$.
\end{abstract}

() 2021 Elsevier B.V. All rights reserved.

\section{Introduction}

Let $\mathcal{A}$ be a unital $\mathrm{C}^{*}$-algebra, $\mathcal{B}$ a sub-C $\mathrm{C}^{*}$-algebra of $\mathcal{A}$ which is a $\mathrm{W}^{*}$-algebra, and $\mathcal{U}_{\mathcal{A}}, \mathcal{U}_{\mathcal{B}}$ its respective unitary groups. Theorem II of [7] or Theorem I-2 of [8] (and the Remark that follows it) imply that for every element $\rho \in \mathcal{U}_{\mathcal{A}} / \mathcal{U}_{\mathcal{B}}$, and every tangent vector $x \in T_{\rho}\left(\mathcal{U}_{\mathcal{A}} / \mathcal{U}_{\mathcal{B}}\right)$, there exists a minimal lift $Z \in \mathcal{A}^{a h}$ of $x(\|Z\| \leqslant\|Z+D\|$ for all $D \in \mathcal{B})$ such that the curves

$$
\gamma(t)=L_{e^{t Z}} \cdot \rho
$$

\footnotetext{
Partially supported by Grants CONICET (PIP 0525), ANPCyT (PICT 20151505 and 2017-2522) and UNRN (PI 40-B-738).

* Corresponding author.

E-mail addresses: tbottazzi@unrn.edu.ar (T. Bottazzi), avarela@campus.ungs.edu.ar (A. Varela).
} 
are all the possible short curves (under a natural distance) starting at $\rho$ with fixed initial velocity $x$, where $L_{u} \cdot \rho$ denotes the left action of $u \in \mathcal{U}_{\mathcal{A}}$ on $\rho$. In this context, we will call this $Z$ a minimal operator.

Moreover in [8] local and global Hopf-Rinow theorems were proved in this context with additional hypothesis on the unitary groups involved.

If the assumption of $\mathcal{B}$ being a von Neumann algebra is relaxed, Theorem I of [7] proves that every minimal lift $Z$ still produces a short curve if $\mathcal{B}$ is only required to be a $\mathrm{C}^{*}$-algebra. Nevertheless, in this case such a $Z$ may not exist for every tangent vector $x$ (see for example the discussion following Proposition 18 in [3] or the properties of $Z_{2}$ defined in (4.2) in this paper). Therefore, if $\mathcal{A}$ and $\mathcal{B}$ are $C^{*}$-algebras the parameterization of minimal curves with arbitrary initial velocity is not known in general nor the existence of geodesic neighborhoods. The main objective of this work is the study of short curves of a particular example where the subalgebra $\mathcal{B}$ is not a von Neumann algebra.

Denote with $\mathcal{K}+\mathbb{C}$ the $\mathrm{C}^{*}$-algebra obtained after the unitization of the compact operators in $\mathcal{B}(\mathcal{H})$, that is $\{X \in \mathcal{B}(\mathcal{H}): X=K+\theta I, K \in \mathcal{K}, \theta \in \mathbb{C}\} . \mathcal{H}$ will be a separable Hilbert space, and $\mathcal{U}_{\mathcal{K}+\mathbb{C}}$ will denote the unitary operators of $\mathcal{K}(\mathcal{H})+\mathbb{C}$. If we fix an orthonormal basis $\left\{e_{i}\right\}_{i \in \mathbb{N}}$ in $\mathcal{H}$, we can consider matricial characterizations of elements in $\mathcal{B}(\mathcal{H})$ as diagonal operators. Let $b$ be a compact diagonal selfadjoint operator with spectral multiplicity one, and $\mathcal{O}_{b}$ the orbit

$$
\mathcal{O}_{b}=\mathcal{O}_{b}^{\mathcal{U}_{\mathcal{K}+\mathbb{C}}}=\left\{u b u^{*}: u \in \mathcal{U}_{\mathcal{K}+\mathbb{C}}\right\}
$$

This orbit has a structure of a smooth homogeneous space under the action of $\mathcal{U}_{\mathcal{K}+\mathbb{C}}$ with the identification $\mathcal{O}_{b} \simeq \mathcal{U}_{\mathcal{K}+\mathbb{C}} / \mathcal{U}_{\mathcal{D}(\mathcal{K}+\mathbb{C})}$ (see for example Lemma 1 of [4] and the discussion that follows it).

As we comment in Remark 3.2 the homogeneous space $\mathcal{O}_{b}$ coincides with the orbit of $b$ under the action of several other unitary subgroups. Moreover, a natural Finsler metric defined on the tangent spaces (see 3.1) and a distance (see (3.6)) in $\mathcal{O}_{b}$ is also preserved if we consider those different unitary subgroups.

In this context, we analyze geodesic neighborhoods of $\mathcal{O}_{b}$ and cases of short curves satisfying initial conditions or connecting given endpoints that cannot be obtained using minimal operators $V \in(\mathcal{K}(\mathcal{H})+\mathbb{C})^{a h}$. In Theorem 4.1 it is shown that short curves in $\mathcal{O}_{b}$ of the form (1.1) can be constructed using minimal operators $Z \in(\mathcal{K}(\mathcal{H})+\mathcal{D}(\mathcal{B}(\mathcal{H})))^{a h}$. These geodesics and a result from [7] allows us to prove in 5.4 a HopfRinow local theorem for $\mathcal{O}_{b}$. We also consider certain types of minimal operators with diagonal belonging to $\mathcal{D}(\mathcal{B}(\mathcal{H})) \backslash \mathcal{D}\left(\mathcal{K}(\mathcal{H})+\mathbb{C}\right.$ ) and construct with them short curves $\gamma$ in $\mathcal{O}_{b}$ (for the distance (3.6)) such that in a fixed neighborhood, there is no curve $\delta$ of the form $\delta(t)=e^{t V} b e^{-t V}$ with $V$ a minimal vector in $(\mathcal{K}+\mathbb{C})^{a h}$ that starts in $b$ and ends in $\gamma(t)$ (see 5.9). This means that there exist geodesics that cannot be obtained using minimal vectors $V$ in $(\mathcal{K}+\mathbb{C})^{a h}$.

In the general context mentioned at the beginning of this section the previous results imply that if $\mathcal{B}$ is only required to be a $\mathrm{C}^{*}$-algebra then, even when Hopf-Rinow type theorems can be obtained, there exist short curves in $\mathcal{U}_{\mathcal{A}} / \mathcal{U}_{\mathcal{B}}$ that cannot be described using minimal elements of $\mathcal{A}$.

\section{Preliminaries}

Let $\mathcal{B}(\mathcal{H})$ be the algebra of bounded operators on a separable Hilbert space $\mathcal{H}$, and $\mathcal{K}(\mathcal{H})$ and $\mathcal{U}(\mathcal{H})$ the compact and unitary operators respectively. If an orthonormal basis $\left\{e_{i}\right\}_{i \in \mathbb{N}}$ is fixed we can consider matricial representations of each $A \in \mathcal{B}(\mathcal{H})$, that is $A=\left(A_{i, j}\right)_{i, j \in \mathbb{N}}=\left(\left\langle A e_{i}, e_{j}\right\rangle\right)_{i, j \in \mathbb{N}}$ and diagonal operators which we denote with $\mathcal{D}(\mathcal{B}(\mathcal{H}))$. Any $D \in \mathcal{D}(\mathcal{B}(\mathcal{H}))$ fulfills $\left\langle D e_{i}, e_{j}\right\rangle=0$ for every $i \neq j$.

With the preceding notation, we define columns (and similarly, rows) of any operator $A \in \mathcal{B}(\mathcal{H}$ ) as $c_{j}(A)=\sum_{i=1}^{\infty}\left\langle A e_{i}, e_{j}\right\rangle e_{j}=\left(A_{1 j}, A_{2 j}, \ldots\right) \subset \ell^{2}$, for each $j \in \mathbb{N}$.

We call $\mathcal{U}_{k}$ the Fredholm subgroup of $\mathcal{U}(\mathcal{H})$, defined as

$$
\begin{aligned}
\mathcal{U}_{k} & =\{u \in \mathcal{U}(\mathcal{H}): u-I \in \mathcal{K}(\mathcal{H})\} \\
& =\left\{u \in \mathcal{U}(\mathcal{H}): \exists K \in \mathcal{K}(\mathcal{H})^{a h}, u=e^{K}\right\},
\end{aligned}
$$


and the subgroup studied in [5]:

$$
\begin{aligned}
\mathcal{U}_{k, d} & =\left\{u \in \mathcal{U}(\mathcal{H}): u-e^{D} \in \mathcal{K}(\mathcal{H}) \text { for } D \in(\mathcal{D}(\mathcal{B}(\mathcal{H})))^{a h}\right\} \\
& =\left\{u \in \mathcal{U}(\mathcal{H}): \exists K \in \mathcal{K}(\mathcal{H})^{a h} \text { and }(\mathcal{D}(\mathcal{B}(\mathcal{H})))^{a h}, \text { such that } u=e^{K} e^{D}\right\}
\end{aligned}
$$

where $I$ is the identity in $\mathcal{B}(\mathcal{H})$ and the superscript ${ }^{a h}$ means anti-Hermitian as well as ${ }^{h}$ means Hermitian.

Consider the unitization of $\mathcal{K}(\mathcal{H})$

$$
\mathcal{K}+\mathbb{C}=\mathcal{K}(\mathcal{H})+\{\lambda I: \lambda \in \mathbb{C}\} \subset \mathcal{B}(\mathcal{H})
$$

endowed with the norm

$$
\|K+\lambda I\|_{\mathcal{K}+\mathbb{C}}=\sup \{\|K C+\lambda C\|: C \in \mathcal{K}(\mathcal{H}),\|C\|=1\},
$$

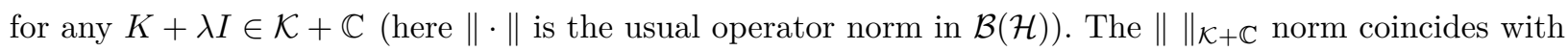
the operator norm in $\mathcal{B}(\mathcal{H})$ :

$$
\|K+\lambda I\|_{\mathcal{K}+\mathbb{C}}=\|K+\lambda I\|
$$

(this follows after considering the multiplication $(K+\lambda I) C$ for $C=h \otimes h \in \mathcal{K}(\mathcal{H})$, with $h \in H$ and $\|h\|=1)$.

The space $\left(\mathcal{K}+\mathbb{C},\|\cdot\|_{\mathcal{K}+\mathbb{C}}\right)$ is a unital $C^{*}$-algebra with unit $I_{\mathcal{K}+\mathbb{C}}=0+1 . I=I$.

Let $\mathcal{D}(\mathcal{K}(\mathcal{H}))=\mathcal{D}(\mathcal{B}(\mathcal{H})) \cap \mathcal{K}(\mathcal{H})$ and define the subspace of diagonal operators of $\mathcal{K}+\mathbb{C}$, given by

$$
\mathcal{D}(\mathcal{K}+\mathbb{C})=\mathcal{D}(\mathcal{K}(\mathcal{H}))+\{\lambda I: \lambda \in \mathbb{C}\}
$$

It is apparent that $\mathcal{D}(\mathcal{K}+\mathbb{C})$ is a unital $C^{*}$-subalgebra of $\mathcal{K}+\mathbb{C}$ and $I_{\mathcal{K}+\mathbb{C}} \in \mathcal{D}(\mathcal{K}+\mathbb{C})$.

If $u=K+\lambda I \in(\mathcal{K}+\mathbb{C})$ is a unitary operator, direct computations show that $K K^{*}=K^{*} K$ and $|\lambda|=1$. Therefore, there exists $\theta \in \mathbb{R}\left(\lambda=e^{i \theta}\right)$ such that $u$ verifies that $u-e^{i \theta I} \in \mathcal{K}(\mathcal{H})$. Then $u \in \mathcal{U}_{k, d}$ (see (2.2)) and therefore there exists $K_{0} \in \mathcal{K}(\mathcal{H})^{a h}$ such that $u=e^{K_{0}} e^{i \theta I}$ for the same $\theta$ (as seen in the proof of Proposition 3.3 in [5]). Moreover, it is apparent that if $u=e^{K} e^{i \theta I}$, with $\theta \in \mathbb{R}$ and $K \in \mathcal{K}(\mathcal{H})^{a h}$, then $u=e^{i \theta I}+\left(\sum_{n \geqslant 1} \frac{K^{n}}{n !} e^{i \theta I}\right) \in \mathcal{U}_{\mathcal{K}+\mathbb{C}}$, the unitary group of $\mathcal{K}+\mathbb{C}$.

Similar considerations can be made with the unitaries of $\mathcal{D}(\mathcal{K}+\mathbb{C})$. If $v \in \mathcal{U}_{\mathcal{D}(\mathcal{K}+\mathbb{C})}$ then $v=d+e^{i \theta I}$ with $d \in \mathcal{D}(\mathcal{K}(\mathcal{H}))$ and $\theta \in \mathbb{R}$. This implies that $\left|d_{j, j}+e^{i \theta}\right|=1$ for all $j \in \mathbb{N}$ and therefore $\left(d+e^{i \theta I}\right)=e^{i R}$ with $R$ a real diagonal matrix such that $R_{j, j} \rightarrow \theta$ when $j \rightarrow \infty$. Conversely, if $v=e^{i R}$ with $R_{j, j} \in \mathbb{R}$ and $\lim _{j \rightarrow \infty} R_{j, j}=\theta$, then $v=e^{i(R-\theta I)} e^{i \theta I} \in \mathcal{U}_{\mathcal{D}(\mathcal{K}+\mathbb{C})}$ because $\lim _{j \rightarrow \infty}\left(R_{j, j}-\theta\right)=0$ and therefore $i(R-\theta I) \in \mathcal{K}(\mathcal{H})^{a h}$.

Then the unitary groups of $\mathcal{K}+\mathbb{C}$ and $\mathcal{D}(\mathcal{K}+\mathbb{C})$ can be described as follows:

$$
\begin{aligned}
\mathcal{U}_{\mathcal{K}+\mathbb{C}} & =\mathcal{U}_{k} \cdot\left\{e^{i \theta} I: \theta \in \mathbb{R}\right\}=\left\{e^{K} e^{i \theta I}: K \in \mathcal{K}(\mathcal{H})^{a h} \text { and } \theta \in \mathbb{R}\right\} \\
& =\left\{e^{K+i \theta I}: K \in \mathcal{K}(\mathcal{H})^{a h} \text { and } \theta \in \mathbb{R}\right\}
\end{aligned}
$$

and

$$
\begin{aligned}
\mathcal{U}_{\mathcal{D}(\mathcal{K}+\mathbb{C})} & =\left\{d+e^{i \theta I}: d \in \mathcal{D}(\mathcal{K}(\mathcal{H})), \theta \in \mathbb{R} \text { such that }\left|d_{j, j}+e^{i \theta I}\right|=1\right\} \\
& =\left\{e^{d_{0}+i \theta I}: d_{0} \in \mathcal{D}(\mathcal{K}(\mathcal{H}))^{a h} \text { and } \theta \in \mathbb{R}\right\} \\
& =\left\{e^{L_{0}}: L_{0} \in \mathcal{D}(\mathcal{B}(\mathcal{H}))^{a h} \text { such that } \lim _{j \rightarrow \infty}\left(L_{0}\right)_{j, j}=i \theta \text { with } \theta \in \mathbb{R}\right\}
\end{aligned}
$$




\section{The homogeneous unitary orbit of a self-adjoint compact operator}

Given a subgroup $\mathcal{U} \subset \mathcal{U}(\mathcal{H})$ we will denote with $\mathcal{O}_{b}^{\mathcal{U}}$ the orbit of self-adjoint element $b \in \mathcal{K}(\mathcal{H})^{h}$ by a subgroup $\mathcal{U} \subset \mathcal{U}(\mathcal{H})$, that is

$$
\mathcal{O}_{b}^{\mathcal{U}}=\left\{u b u^{*}: u \in \mathcal{U}\right\}
$$

Let $b=\operatorname{Diag}\left(\left\{b_{i}\right\}_{i \in \mathbb{N}}\right) \in \mathcal{D}\left(\mathcal{K}(\mathcal{H})^{h}\right)$ denote the diagonal operator with the sequence $\left\{b_{i}\right\}_{i \in \mathbb{N}}$ in its diagonal.

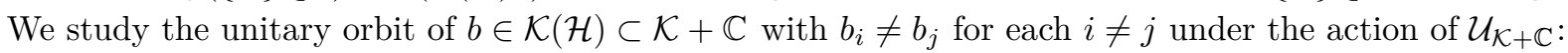

$$
\mathcal{O}_{b}=\mathcal{O}_{b}^{\mathcal{U}_{\mathcal{K}+\mathbb{C}}}=\left\{u b u^{*}: u \in \mathcal{U}_{\mathcal{K}+\mathbb{C}}\right\} .
$$

Observe that it is apparent that the following inclusions hold for these subgroups of $\mathcal{U}(\mathcal{H})$ :

$$
\mathcal{U}_{k} \subsetneq \mathcal{U}_{\mathcal{K}+\mathbb{C} \subsetneq \mathcal{U}_{k, d}}
$$

Nevertheless the orbit of $b$ under the three subgroups is the same set $\mathcal{O}_{b}$ because

$$
e^{K} b e^{-K}=e^{K} e^{i t I} b e^{-i t I} e^{-K}=e^{K+i t I} b e^{-K-i t I}
$$

for $t \in \mathbb{R}$ and then $\mathcal{O}_{b}^{\mathcal{U}_{k}}=\mathcal{O}_{b}^{\mathcal{U}_{k, d}}$ (see for example Remark 4.4 in [5]). Moreover, we will show further that the three of them share the same natural Finsler metric on the tangent spaces (as seen in Remark 4.5 in [5] for the cases of $\mathcal{O}_{b}^{\mathcal{U}_{k}}$ and $\mathcal{O}_{b}^{\mathcal{U}_{k, d}}$ ).

$\mathcal{O}_{b}$ has a smooth structure as described in Lemma 1 in [4] and the comments that follow it.

The isotropy group at any $c \in \mathcal{O}_{b}=\mathcal{O}_{b}^{\mathcal{U}_{\mathcal{K}+\mathbb{C}}}$ is $\mathcal{I}_{c}=\left\{u \in \mathcal{U}_{\mathcal{K}+\mathbb{C}}: u c u^{*}=c\right\}$. In particular,

$$
\mathcal{I}_{b}=\left\{u \in \mathcal{U}_{\mathcal{K}+\mathbb{C}}:[u, b]=0\right\}=\mathcal{U}_{\mathcal{D}(\mathcal{K}+\mathbb{C})}
$$

Remark 3.1. If $c \in \mathcal{O}_{b}$, the following identification can be made:

$$
T_{c} \mathcal{O}_{b} \cong\left(T \mathcal{U}_{\mathcal{K}+\mathbb{C}}\right)_{1} /\left(T \mathcal{I}_{b}\right)_{1}=(\mathcal{K}+\mathbb{C})^{a h} /(\mathcal{D}(\mathcal{K}+\mathbb{C}))^{a h}
$$

Observe that

$$
(\mathcal{K}+\mathbb{C})^{a h} / \mathcal{D}(\mathcal{K}+\mathbb{C})^{a h}=\left\{[X]: X=K_{0}+i \theta_{0} I, K_{0} \in \mathcal{K}(\mathcal{H})^{a h} \text { and } \theta_{0} \in \mathbb{R}\right\},
$$

where $[X]$ is the class defined as $Y \in[X]$ iff $Y=X+d+i \theta I$ for $d \in \mathcal{D}\left(\mathcal{K}(\mathcal{H})^{a h}\right)$ and $\theta \in \mathbb{R}$. This quotient space is endowed with the usual quotient norm, that in this case for $X=K_{0}+i \theta_{0} I$ is

$$
\begin{aligned}
\|[X]\|=\left\|\left[K_{0}+i \theta_{0} I\right]\right\| & =\inf _{\theta \in \mathbb{R} ; d \in \mathcal{D}\left(\mathcal{K}(\mathcal{H})^{a h}\right)}\left\|K_{0}+i \theta_{0} I+d+i \theta I\right\|_{\mathcal{K}+\mathbb{C}} \\
& =\inf _{\theta \in \mathbb{R} ; d \in \mathcal{D}\left(\mathcal{K}(\mathcal{H})^{a h}\right)}\left\|K_{0}+d+i \theta I\right\|_{\mathcal{K}+\mathbb{C} .}
\end{aligned}
$$

In this context, a natural Finsler metric for any $x \in T_{b} \mathcal{O}_{b}, x=X b-b X$, with $X \in(\mathcal{K}+\mathbb{C})^{a h}$ is

$$
\begin{aligned}
\|x\|_{b} & =\inf \left\{\|Y\|: Y \in(\mathcal{K}+\mathbb{C})^{a h}, Y b-b Y=X b-b X\right\} \\
& =\inf _{\substack{d \in \mathcal{D}\left(\mathcal{K}(\mathcal{H})^{a h}\right) \\
\theta \in \mathbb{R}}}\|X+d+i \theta I\|_{\mathcal{K}+\mathbb{C}}=\inf _{\substack{d \in \mathcal{D}\left(\mathcal{K}(\mathcal{H})^{a h}\right) \\
\theta \in \mathbb{R}}}\|X+d+i \theta I\|
\end{aligned}
$$


where $X+d+i \theta I$ is any element of the class $[X]=\left\{Y \in(\mathcal{K}+\mathbb{C})^{a h}: Y=X+d+i \theta I\right.$, for $\theta \in \mathbb{R}$ and $d \in$ $\left.\mathcal{D}\left(\mathcal{K}(\mathcal{H})^{a h}\right)\right\}$ in $(\mathcal{K}+\mathbb{C})^{a h} / \mathcal{D}(\mathcal{K}+\mathbb{C})^{a h}$.

An element $Y \in \mathcal{B}(\mathcal{H})^{a h}$ such that $Y b-b Y=x$ and $\|Y\|_{\mathcal{K}+\mathbb{C}}=\|Y\|=\|[X]\|=\|x\|_{b}$ will be called a minimal lifting for $x$, and its diagonal will be a minimal diagonal approximant (or minimizing diagonal) for $Y$. This operator $Y$ may not be compact nor unique (see [3]), and it will be called a minimal operator.

Given any $c=e^{K+i t I} b e^{-K-i t I}=e^{K} b e^{-K} \in \mathcal{O}_{b}\left(K \in \mathcal{K}(\mathcal{H})^{a h}, t \in \mathbb{R}\right)$ we can define the norm in its tangent space $T_{c} \mathcal{O}_{b}$ using that $z=Z c-c Z \in T_{c} \mathcal{O}_{b}$ for $Z=e^{K} X e^{-K} \in \mathcal{K}(\mathcal{H})^{a h}$ and $X b-b X \in T_{b} \mathcal{O}_{b}$. Then the Finsler norm in $T_{c} \mathcal{O}_{b}$ is $\|z\|_{c}=\|[Z]\|=\inf \left\{\|Y\|: Y \in(\mathcal{K}+\mathbb{C})^{a h}, Y c-c Y=Z c-c Z\right\}=\|[X]\|$. Note that this norm is invariant under the action of $\mathcal{U}_{\mathcal{K}+\mathbb{C}}$.

Remark 3.2. As it was mentioned before in (3.2), the following orbits are equal

$$
\mathcal{O}_{b}^{\mathcal{U}_{\mathcal{K}+\mathbb{C}}}=\mathcal{O}_{b}^{\mathcal{U}_{k}}=\mathcal{O}_{b}^{\mathcal{U}_{k, d}}
$$

for $\mathcal{U}_{k} \subsetneq \mathcal{U}_{\mathcal{K}+\mathbb{C}} \subsetneq \mathcal{U}_{k, d}$ defined in (2.1), (2.3) and (2.2) respectively (see Proposition 4.1, 4.4 and Remark 4.7 in $[5])$.

Let $X \in(\mathcal{K}+\mathbb{C})^{a h}, X=K+i t I$ with $K \in \mathcal{K}(\mathcal{H})^{a h}$ and $t \in \mathbb{R}$, then

$$
\begin{aligned}
\inf _{D \in \mathcal{D}\left(\mathcal{B}(\mathcal{H})^{a h}\right)}\|K+D\| & =\inf _{D \in \mathcal{D}\left(\mathcal{B}(\mathcal{H})^{a h}\right)}\|\overbrace{K+i t I}^{X}+D\| \leqslant \inf _{d \in \mathcal{D}\left(\mathcal{K}(\mathcal{H})^{a h}\right)}\|X+i \theta I+d\| \\
& =\inf _{\substack{\theta \in \mathcal{R}\left(\mathcal{K}(\mathcal{H})^{a h}\right) \\
\theta \in \mathbb{R}}}\|K+i \theta I+d\| \leqslant \inf _{d \in \mathcal{D}\left(\mathcal{K}(\mathcal{H})^{a h}\right)}\|K+d\| .
\end{aligned}
$$

But since $\inf _{d \in \mathcal{D}\left(\mathcal{K}(\mathcal{H})^{a h}\right)}\|K+d\|=\inf _{D \in \mathcal{D}\left(\mathcal{B}(\mathcal{H})^{a h}\right)}\|K+D\|$, [Prop. 5, [3]], the previous inequalities imply that all those infimums are equal.

This means that also the Finsler metric defined for $T_{c} \mathcal{O}_{b}$ in (3.3) coincides if we consider any of the quotients $\mathcal{K}(\mathcal{H})^{a h} / \mathcal{D}\left(\mathcal{K}(\mathcal{H})^{a h}\right),(\mathcal{K}+\mathbb{C})^{a h} / \mathcal{D}(\mathcal{K}+\mathbb{C})^{a h}$ or $\left(\mathcal{K}(\mathcal{H})^{a h}+\mathcal{D}(\mathcal{B}(\mathcal{H}))^{a h}\right) / \mathcal{D}(\mathcal{B}(\mathcal{H}))^{a h}$ (see also Remark 4.5 in [5]).

Remark 3.3. Observe that nevertheless $\mathcal{O}_{b} \subsetneq \mathcal{O}_{b}^{\mathcal{U}(\mathcal{H})}$. This follows because if we suppose that for every $X \in \mathcal{B}(\mathcal{H})^{a h}$ holds that $e^{X} b e^{-X}=e^{K} b e^{-K}$ for $K \in \mathcal{K}(\mathcal{H})^{a h}$, then $e^{-K} e^{X}$ must be a diagonal unitary. Therefore, for all $X \in \mathcal{B}(\mathcal{H})^{a h}$ we could write that $e^{X}=e^{K} e^{D}$ for $D \in \mathcal{D}(\mathcal{B}(\mathcal{H}))^{a h}$ which is known to be false (see for example Remark 3.7 in [5]).

Consider piecewise smooth curves $\beta:[a, b] \rightarrow \mathcal{O}_{b}^{\mathcal{U}_{\mathcal{K}+\mathbb{C}}}$. We define

$$
\begin{gathered}
\mathrm{L}(\beta)=\int_{a}^{b}\left\|\beta^{\prime}(t)\right\|_{\beta(t)} d t, \text { and } \\
\operatorname{dist}\left(c_{1}, c_{2}\right)=\inf \left\{\mathrm{L}(\beta): \beta \text { is smooth, } \beta(a)=c_{1}, \beta(b)=c_{2}\right\}
\end{gathered}
$$

as the rectifiable length of $\beta$ and distance between two points $c_{1}, c_{2} \in \mathcal{O}_{b}^{\mathcal{U}_{\mathcal{K}+\mathbb{C}}}$, respectively. 


\section{A short curve in $\mathcal{O}_{b}$ obtained acting with a minimal operator not belonging to $(\mathcal{K}+\mathbb{C})^{a h} / \mathcal{D}(\mathcal{K}+\mathbb{C})^{a h}$}

Consider the following operator described as an infinite matrix:

$$
Z_{\delta, \gamma}=i\left(\begin{array}{cccccccc}
0 & -\delta & \gamma & -\delta^{2} & \gamma^{2} & -\delta^{3} & \gamma^{3} & \ldots \\
-\delta & 0 & \gamma & -\delta^{2} & \gamma^{2} & -\delta^{3} & \gamma^{3} & \ldots \\
\gamma & \gamma & 0 & -\delta^{2} & \gamma^{2} & -\delta^{3} & \gamma^{3} & \ldots \\
-\delta^{2} & -\delta^{2} & -\delta^{2} & 0 & \gamma^{2} & -\delta^{3} & \gamma^{3} & \ldots \\
\gamma^{2} & \gamma^{2} & \gamma^{2} & \gamma^{2} & 0 & -\delta^{3} & \gamma^{3} & \ldots \\
-\delta^{3} & -\delta^{3} & -\delta^{3} & -\delta^{3} & -\delta^{3} & 0 & \gamma^{3} & \ldots \\
\gamma^{3} & \gamma^{3} & \gamma^{3} & \gamma^{3} & \gamma^{3} & \gamma^{3} & 0 & \ldots \\
\vdots & \vdots & \vdots & \vdots & \vdots & \vdots & \vdots & \ddots
\end{array}\right) \text {, with } \gamma, \delta \in(0,1)
$$

$Z_{\delta, \gamma}$ is a Hilbert-Schmidt operator which has been studied in [4] in its self-adjoint version. We recall here some of its properties.

Let $\gamma^{2}=\delta$ and $\delta^{2}<\gamma$ (for example $\gamma=1 / 2$ and $\delta=1 / 4$ ), and denote with $Z_{\delta, \gamma}^{[1]}$ the operator defined by the matrix of $Z_{\delta, \gamma}$ with zeros in the first column and row, with $c_{1}\left(Z_{\delta, \gamma}\right)$ the first column of $Z_{\delta, \gamma}$, and with $D_{0}$ the (uniquely determined) diagonal matrix such that every $\left(D_{0}\right)_{i, i}$ is chosen to satisfy $c_{i}\left(Z_{\delta, \gamma}\right) \perp c_{1}\left(Z_{\delta, \gamma}\right)$ for all $i \neq 1$. Then

$$
Z_{o}=\frac{\left\|Z_{\delta, \gamma}^{[1]}+D_{0}\right\|}{\left\|c_{1}\left(Z_{\delta, \gamma}\right)\right\|}\left(Z_{\delta, \gamma}-Z_{\delta, \gamma}^{[1]}\right)+Z_{\delta, \gamma}^{[1]}
$$

is also a Hilbert-Schmidt operator with the property that $D_{0}$ (constructed as mentioned before) is a minimal diagonal for $Z_{o}$ (see (5.1) in [4] for a detailed proof of these statements and the following comments). Moreover, it has been proved that $D_{0} \in \mathcal{D}\left(\mathcal{B}(\mathcal{H})^{a h}\right)$ is the unique bounded best approximant anti-Hermitian diagonal of $Z_{o}$. $D_{0}$ has the particular property that $\lim _{k \rightarrow \infty}\left(D_{0}\right)_{2 k, 2 k} \neq \lim _{k \rightarrow \infty}\left(D_{0}\right)_{2 k+1,2 k+1}$ and both limits are not null. Therefore $D_{0}$ is not compact and we call it an oscillant diagonal. We will write with

$$
Z_{2}=Z_{o}+D_{0}
$$

to denote the minimal operator constructed as above.

Then

$$
\operatorname{dist}\left(Z_{o}, \mathcal{D}\left(\mathcal{K}(\mathcal{H})^{a h}\right)\right)=\left\|\left[Z_{o}\right]\right\|_{\mathcal{K}(\mathcal{H})^{a h} / \mathcal{D}\left(\mathcal{K}(\mathcal{H})^{a h}\right)}=\left\|Z_{2}\right\|=\left\|c_{1}\left(Z_{2}\right)\right\|=\left\|c_{1}\left(Z_{o}\right)\right\|
$$

Theorem 4.1. Let $b=\operatorname{Diag}\left(\left\{b_{i}\right\}_{i \in \mathbb{N}}\right) \in \mathcal{D}\left(\mathcal{K}(\mathcal{H})^{h}\right)$ with $b_{i} \neq b_{j}$ for each $i \neq j$. Consider the unitary orbit $\mathcal{O}_{b}^{\mathcal{U}_{\mathcal{K}+\mathbb{C}}}$ defined in (3.1) and $x=Z b-b Z \in T_{b} \mathcal{O}_{b}$, for $Z$ a minimal operator in $\mathcal{K}(\mathcal{H})^{a h}+\mathcal{D}(\mathcal{B}(\mathcal{H}))^{a h}$. Then the uniparametric group curve $\gamma(t)=e^{t Z}$ be ${ }^{-t Z}$ has minimal length in the class of all curves in $\mathcal{O}_{b}^{\mathcal{U}_{\mathcal{K}+\mathbb{C}}}$ joining $\gamma(0)$ and $\gamma(t)$ for each $t \in\left[-\frac{\pi}{2\|Z\|}, \frac{\pi}{2\|Z\|}\right]$.

Proof. This proof is a direct consequence of mentioned previous results, but we include here the citations and reasonings for the sake of clarity.

By Theorem 4.2 in [5], $\gamma(t) \in \mathcal{O}_{b}^{\mathcal{U}_{k}}$ for any $t \in \mathbb{R}$. Using Remark 3.2, we obtain that $\gamma(t) \in \mathcal{O}_{b}^{\mathcal{U}_{\mathcal{K}+\mathbb{C}}=}$ $\mathcal{O}_{b}, \forall t$. Moreover,

$$
\|x\|_{b}=\|Z b-b Z\|_{b}=\|[Z]\|=\inf _{\theta \in \mathbb{R} ; d \in \mathcal{D}\left(\mathcal{K}(\mathcal{H})^{a h}\right)}\|Z+d+i \theta I\|=\|Z\|,
$$


where the minimality of $Z$ implies the last equality.

Consider $\mathcal{P}_{b}=\left\{u b u^{*}: u \in \mathcal{U}(\mathcal{H})\right\}$, then by Theorem II in [7], since $Z$ is minimal, the curve $\gamma$ has minimal length over all the smooth curves in $\mathcal{P}_{b}$ that join $\gamma(0)=b$ and $\gamma(t)$, with $|t| \leqslant \frac{\pi}{2\|Z\|}$. Since clearly $\mathcal{O}_{b}^{\mathcal{U}_{\mathcal{K}+\mathbb{C}}} \subseteq \mathcal{P}_{b}$, then for each $t_{0} \in\left[-\frac{\pi}{2\|Z\|}, \frac{\pi}{2\|Z\|}\right]$ follows that $\gamma$ is a short curve in $\mathcal{O}_{b}^{\mathcal{U}_{\mathcal{K}+\mathbb{C}}}$, that is

$$
\mathrm{L}\left(\left.\gamma\right|_{\left[0, t_{0}\right]}\right)=\operatorname{dist}\left(b, \gamma\left(t_{0}\right)\right)
$$

where $\operatorname{dist}\left(b, \gamma\left(t_{0}\right)\right)$ is the rectifiable distance between $b$ and $\gamma\left(t_{0}\right)$ defined in (3.6).

Corollary 4.2. Let $b=\operatorname{Diag}\left(\left\{b_{i}\right\}_{i \in \mathbb{N}}\right) \in \mathcal{D}\left(\mathcal{K}(\mathcal{H})^{h}\right)$ with $b_{i} \neq b_{j}$ for each $i \neq j$. Consider the unitary orbit $\mathcal{O}_{b}^{\mathcal{U}_{\mathcal{K}+\mathbb{C}}}$ defined in (3.1), $x=Z_{o} b-b Z_{o} \in T_{b} \mathcal{O}_{b}$, for $Z_{o}$ defined in (4.1), with $D_{0}$ its unique minimizing diagonal, and $Z_{2}=Z_{o}+D_{0}$ defined in (4.2).

Then the uniparametric group curve $\gamma(t)=e^{t Z_{2}} b e^{-t Z_{2}}$ has minimal length in the class of all curves in $\mathcal{O}_{b}^{\mathcal{U}_{\mathcal{K}+\mathbb{C}}}$ joining $\gamma(0)$ and $\gamma(t)$ for each $t \in\left[-\frac{\pi}{2\left\|Z_{2}\right\|}, \frac{\pi}{2\left\|Z_{2}\right\|}\right]$.

Proof. If we consider $Z=Z_{2}=Z_{o}+D_{0}$ in the statements of Theorem 4.1, then $Z_{2}$ satisfies the conditions required and therefore the proof is apparent.

The previous result will allow us to state that the converse of Theorem I in [7] does not necessary hold when the subalgebra considered (here $\mathcal{D}(\mathcal{K}+\mathbb{C})$ ) is not a von Neumann algebra. Let us describe the context of that article. Let $\mathcal{A}$ be a $\mathrm{C}^{*}$-algebra and $\mathcal{B}$ a $\mathrm{C}^{*}$-subalgebra, then a natural Finsler metric as the one in (3.3) is defined for the generalized flag $\mathcal{P}=\mathcal{U}_{\mathcal{A}} / \mathcal{U}_{\mathcal{B}}$. If the element $X \in \mathcal{A}^{a h}$ is minimal for a tangent vector $x \in T_{p}\left(\mathcal{U}_{\mathcal{A}} / \mathcal{U}_{\mathcal{B}}\right) \simeq T_{1}\left(\mathcal{U}_{\mathcal{A}}\right) / T_{1}\left(\mathcal{U}_{\mathcal{B}}\right)\left(\right.$ that is: $x=X p-p X$ and $\left.\|X\|=\inf \left\{\|Y\|: Y \in \mathcal{A}^{a h} ; Y p-p Y=x\right\}\right)$ then the curve $\gamma(t)=L_{e^{t X}} \cdot p$ has minimal length for $|t| \leqslant \frac{\pi}{2\|X\|}($ where $L$ is a left action on $\mathcal{P}$ ) with the distance defined in (3.6).

The following result shows that there might exist some minimal curves $\gamma$ in these generalized flags $\mathcal{P}=\mathcal{U}_{\mathcal{K}+\mathbb{C}} / \mathcal{U}_{\mathcal{D}(\mathcal{K}+\mathbb{C})}$ that are not of the form $\gamma(t)=L_{e^{t Z}} \cdot p$ for $Z \in \mathcal{A}^{a h}$ a minimal lifting of a tangent vector $x \in T_{1}\left(\mathcal{U}_{\mathcal{A}}\right) / T_{1}\left(\mathcal{U}_{\mathcal{B}}\right)$.

Remark 4.3. Let $Z_{2}$ be the operator defined in (4.2). As it was mentioned in Corollary 4.2, the uniparametric curve $\gamma(t)=e^{t Z_{2}} b e^{-t Z_{2}}$ has minimal length in the class of all curves in $\mathcal{O}_{b}=\mathcal{O}_{b}^{\mathcal{U}_{\mathcal{K}+\mathbb{C}}}$ joining $\gamma(0)$ and $\gamma(t)$ for each $t \in\left[-\frac{\pi}{2\left\|Z_{2}\right\|}, \frac{\pi}{2\left\|Z_{2}\right\|}\right]$.

Therefore the curve $\gamma$ is included in $\mathcal{O}_{b}$ with initial conditions $\gamma(0)=b, \gamma^{\prime}(0)=x=Z_{o} b-b Z_{o}$ even for velocity vectors $x \in T_{b} \mathcal{O}_{b}$ that do not have a minimal compact lifting $K_{0}$ (recall that $Z_{o}+D_{0}$ is not compact and $D_{0}$ is its unique minimizing diagonal). Thus $\mathcal{U}_{\mathcal{K}+\mathbb{C}}$ is an example of a group whose action on $\mathcal{O}_{b}$ has short curves that might not be described using minimal vectors $Y \in(\mathcal{K}+\mathbb{C})^{a h}$. This is not new (for instance, see Remark 4.7 in [5]), but in the present case $\mathcal{K}+\mathbb{C}$ and $\mathcal{D}(\mathcal{K}+\mathbb{C}$ ), whose anti-Hermitian elements are the Lie-algebras of $\mathcal{U}_{\mathcal{K}+\mathbb{C}}$ and $\mathcal{U}_{\mathcal{D}(\mathcal{K}+\mathbb{C})}$ respectively, are unital $C^{*}$-algebras.

We will develop some details of this situation in the next section.

\section{Neighborhoods of short curves defined by minimal vectors in $\mathcal{U}_{\mathcal{K}+\mathbb{C}} / \mathcal{U}_{\mathcal{D}(\mathcal{K}+\mathbb{C})}$}

In this section we will consider the problem of the existence of a neighborhood around $b \in \operatorname{Diag}(\mathcal{K}(\mathcal{H}))^{h}$ with $b_{i, i} \neq b_{j, j}$ for $i \neq j$ whose elements can be joined with $b$ with a short curve of the form

$$
\gamma(t)=e^{t Z} b e^{-t Z}
$$

for some minimal anti-Hermitian element $Z \in(\mathcal{K}+\mathbb{C})^{a h}$ and $t$ in some interval. 
Recall here $Z_{2}=Z_{o}+D_{0}$ defined in (4.2) where $Z_{o} \in \mathcal{K}(\mathcal{H})^{a h}$ is the Hilbert-Schmidt operator defined in (4.1) and $D_{0}$ is its unique minimizing diagonal with the property that $D_{0}$ has subsequences that converge to two different (not null) limits as described in the previous section. Moreover, $Z_{2}$ satisfies,

(1) $\left\|Z_{2}\right\|=\left\|c_{1}\left(Z_{2}\right)\right\|$

(2) $c_{1}\left(Z_{2}\right)_{1}=\left(Z_{2}\right)_{1,1}=0$ and

(3) $c_{1}\left(Z_{2}\right)_{j}=\left(Z_{2}\right)_{j, 1} \neq 0$ for all $j \neq 1$.

Lemma 5.1. Let $\gamma(t)=e^{t Z} b e^{-t Z} \subset \mathcal{O}_{b}$ with $Z \in \mathcal{B}(\mathcal{H})^{a h}$ a minimal operator with unique minimizing diagonal, and consider a curve $\delta(t)=e^{t V} e^{-t V}$, with $V \in \mathcal{B}(\mathcal{H})^{\text {ah }}$ another minimal operator such that $\delta^{\prime}(0)=V b-b V=\gamma^{\prime}(0)=Z b-b Z$. Then it must be $Z=V$.

Proof. Since $\delta^{\prime}(0)=\gamma^{\prime}(0)$, then

$$
\delta^{\prime}(0)=V b-b V=\gamma^{\prime}(0)=Z b-b Z
$$

and therefore $V-Z$ commutes with $b$. Then $V-Z \in \mathcal{D}\left(\mathcal{B}(\mathcal{H})^{a h}\right)$ which implies that $Z$ and $V$ must be equal outside their diagonals. Then, $\operatorname{since} \operatorname{Diag}(Z)$ is the only minimizing diagonal for $Z$ and $V$ is also a minimal operator then $\operatorname{Diag}(V)=\operatorname{Diag}(Z)$ which implies that $V=Z$.

Remark 5.2. Observe that if we apply the previous lemma to the case where $Z=Z_{2}$ defined in (4.2) and $\delta$ and $V$ satisfy the assumptions of the lemma, then in particular $V \notin(\mathcal{K}+\mathbb{C})^{a h}$. This is a direct consequence of the fact that $Z_{2}$ has two different non zero diagonal limits, something that $V \in(\mathcal{K}+\mathbb{C})$ cannot satisfy.

Lemma 5.3. Let $Z=K_{Z}+\operatorname{Diag}(Z)$ with $K_{Z} \in \mathcal{K}(\mathcal{H})^{a h}, \operatorname{Diag}(Z) \in \mathcal{D}(\mathcal{B}(\mathcal{H}))^{\text {ah }}$ be a minimal operator and $\gamma:\left[0, \frac{\pi}{2\|Z\|}\right] \rightarrow \mathcal{O}_{b}$ the short curve defined as $\gamma(t)=e^{t Z}$ be $-t Z$ (see Theorem 4.1) and let $\delta:\left[0, \frac{\pi}{2\|V\|}\right] \rightarrow \mathcal{O}_{b}$ be another short curve defined by $\delta(s)=e^{s V} b e^{-s V}$ for $V=K_{V}+\operatorname{Diag}(V)$ another minimal operator with $K_{V} \in \mathcal{K}(\mathcal{H})^{a h}$ and $\operatorname{Diag}(V) \in \mathcal{D}(\mathcal{B}(\mathcal{H}))^{a h}$. Moreover, suppose that there exists $t_{1} \in\left(0, \frac{\log (2) / 8}{\|Z\|}\right]$ and $s_{1} \in\left(0, \frac{\pi}{2\|V\|}\right]$ such that $\gamma\left(t_{1}\right)=\delta\left(s_{1}\right)$.

Then

$$
e^{t_{1} Z}=e^{s_{1} V} e^{-\operatorname{Diag}\left(s_{1} V\right)+\operatorname{Diag}\left(t_{1} Z\right)} \text { and }\left\|s_{1} V\right\|=\left\|t_{1} Z\right\| .
$$

Proof. Note that $t_{1}$ satisfies $\left\|t_{1} Z\right\|<(\log 2) / 8$, and then $t_{1} Z$ is sufficiently close to zero in the sense of Definition 2.1 of [5].

Now consider the equality $\gamma\left(t_{1}\right)=\delta\left(s_{1}\right)$

$$
e^{s_{1} V} b e^{-s_{1} V}=e^{t_{1} Z} b e^{-t_{1} Z} .
$$

Then

$$
b e^{-s_{1} V} e^{t_{1} Z}=e^{-s_{1} V} e^{t_{1} Z} b
$$

which implies that $b$ commutes with $e^{-s_{1} V} e^{t_{1} Z}$. Therefore $e^{-s_{1} V} e^{t_{1} Z}$ is diagonal and unitary. Then there exists $D \in \mathcal{D}(\mathcal{B}(\mathcal{H}))^{a h}$ such that $e^{-s_{1} V} e^{t_{1} Z}=e^{D}$.

Observe that since $Z$ is a minimal operator the length of $\gamma$ restricted to $\left[0, t_{1}\right]$ is $\left\|t_{1} Z\right\|$ and if $\delta$ is a short curve then the length of $\delta$ must coincide with $\left\|t_{1} Z\right\|$ (see Theorem 4.1 in [7]). Since the length of $\delta$ restricted to $\left[0, s_{1}\right]$ equals $\left\|s_{1} V\right\|$ because $V$ is a minimal operator, then $\left\|t_{1} Z\right\|=\left\|s_{1} V\right\|$. Also $t_{1} Z$ is sufficiently close to zero (thus $s_{1} V$ ), so we can apply Proposition 3.11 and Corollary 3.12 of [5] to obtain 


$$
\begin{aligned}
e^{D} & =e^{-s_{1} V} e^{t_{1} Z} \\
& =e^{-s_{1} V+\operatorname{Diag}\left(s_{1} V\right)-\operatorname{Diag}\left(s_{1} V\right)} e^{t_{1} Z-\operatorname{Diag}\left(t_{1} Z\right)+\operatorname{Diag}\left(t_{1} Z\right)} \\
& =e^{K-\operatorname{Diag}\left(s_{1} V\right)+\operatorname{Diag}\left(t_{1} Z\right)}
\end{aligned}
$$

for $K \in \mathcal{K}(\mathcal{H})^{a h}$ with $\operatorname{Diag}(K)=0$. Then, since $\left\|t_{1} Z\right\|=\left\|s_{1} V\right\|<(\log 2) / 8$, then $\|D\|=$ $\left\|\log \left(e^{-s_{1} V} e^{t_{1} Z}\right)\right\| \leqslant-1 / 2 \log \left(2-e^{2\left\|t_{1} Z\right\|+2\left\|s_{1} V\right\|}\right)<\pi$ (see some of the Baker-Campbell-Hausdorff series bounds in [2] or [10]). This implies that $D=K-\operatorname{Diag}\left(s_{1} V\right)+\operatorname{Diag}\left(t_{1} Z\right)$ because $e^{D}=$ $e^{K-\operatorname{Diag}\left(s_{1} V\right)+\operatorname{Diag}\left(t_{1} Z\right)}$ and both anti-Hermitian exponents have norm less than $\pi$ (see for example Corollary 4.2 iii) of [6]). But, $\operatorname{since} \operatorname{Diag}(K)=0$ and $D \in \mathcal{D}(\mathcal{B}(\mathcal{H}))^{a h}$, then

$$
D=-\operatorname{Diag}\left(s_{1} V\right)+\operatorname{Diag}\left(t_{1} Z\right)
$$

and $K=0$.

Theorem 5.4. (Local Hopf-Rinow theorem) There exists $\mathcal{W}_{b} \subset \mathcal{O}_{b}=\mathcal{O}_{b}^{\mathcal{U}_{\mathcal{K}+\mathbb{C}}}$ a neighborhood (with the distance defined in (3.6)) of $b \in \mathcal{D}\left(\mathcal{K}(\mathcal{H})^{h}\right)$ with $b_{i, i} \neq b_{j, j}$ for $i \neq j$, such that for every $\rho \in \mathcal{W}_{b}$ there exists a short curve $\gamma$ in $\mathcal{O}_{b}$ that joins $b$ with $\rho$, and $\gamma:[0,1] \rightarrow \mathcal{W}_{b} \subset \mathcal{O}_{b}$,

$$
\gamma(t)=e^{t\left(K_{\rho}+D_{\rho}\right)} b e^{-t\left(K_{\rho}+D_{\rho}\right)}
$$

with $K_{\rho} \in \mathcal{K}(\mathcal{H})^{a h}, D_{\rho} \in \mathcal{D}(\mathcal{B}(\mathcal{H}))^{a h},\left\|K_{\rho}\right\|,\left\|D_{\rho}\right\|,\left\|K_{\rho}+D_{\rho}\right\|<\frac{\log (2)}{4}$, and $\left(K_{\rho}+D_{\rho}\right)$ a minimal operator in $\mathcal{K}(\mathcal{H})^{a h}+\mathcal{D}(\mathcal{B}(\mathcal{H}))^{a h}$.

Proof. If we consider the isotropy compact generalized flag manifold $\mathcal{P}=\mathcal{U}(\mathcal{H}) / \mathcal{D}\left(\mathcal{U}(\mathcal{H})\right.$ ) then for $\rho_{0} \in \mathcal{P}$ there exists a neighborhood

$$
\mathcal{V}_{\rho_{0}}=\left\{L_{u} \rho_{0}: u=e^{X}, \text { for } X \in \mathcal{B}(\mathcal{H})^{a h},\|X\|<\pi / 2\right\}
$$

where a local Hopf-Rinow theorem holds (see Theorem II-1 and Example 1 of [8]). That is, for every $\rho \in \mathcal{V}_{\rho_{0}}$ there exist a minimal operator $X \in \mathcal{B}(\mathcal{H})^{a h}$ with $\|X\|<\pi / 2$ and a minimal uniparametric group curve $\gamma:[0,1] \rightarrow \mathcal{P}, \gamma(t)=L_{e^{t X}} \rho_{0}$ joining $\gamma(0)=\rho_{0}$ and $\gamma(1)=\rho$.

The generalized flag manifold $\mathcal{P}=\mathcal{U}(\mathcal{H}) / \mathcal{D}(\mathcal{U}(\mathcal{H}))$ can be identified with the unitary orbit of $b \in$ $\mathcal{D}\left(\mathcal{K}(\mathcal{H})^{h}\right)$ with $b_{i, i} \neq b_{j, j}$ as well as its tangent spaces as we have done with $\mathcal{O}_{b}$ in Remark 3.1:

$$
T_{c} \mathcal{O}_{b}^{\mathcal{U}(\mathcal{H})} \simeq T_{1} \mathcal{U}(\mathcal{H}) / T_{1} \mathcal{D}(\mathcal{U}(\mathcal{H}))=\mathcal{B}(\mathcal{H})^{a h} / \mathcal{D}(\mathcal{B}(\mathcal{H}))^{a h}
$$

Since we are using the adjoint action $L$, then $L_{u} b=u b u^{*}$ in this context. And if we consider $\rho_{0}=b$ we can conclude that for any $\rho=e^{K} b e^{-K} \in\left(\mathcal{O}_{b} \cap \mathcal{V}_{b}\right)$ with $K \in \mathcal{K}(\mathcal{H})^{a h}$ (see (3.2)), there exists a minimal operator $Z \in \mathcal{B}(\mathcal{H})^{a h}$ with $\|Z\|<\pi / 2$, such that

$$
\gamma:[0,1] \rightarrow \mathcal{O}_{b}^{\mathcal{U}(\mathcal{H})}, \gamma(t)=e^{t Z} b e^{-t Z}, \text { with } \gamma(0)=\rho_{0}=b \text { and } \gamma(1)=\rho=e^{K} b e^{-K}=e^{Z} b e^{-Z}
$$

Note that in this case $e^{Z}$ cannot be any element of $\mathcal{U}(\mathcal{H})$ because $e^{K} b e^{-K}=e^{Z} b e^{-Z}$ implies that $e^{Z}=e^{K} e^{D}$ for $D \in \mathcal{D}(\mathcal{B}(\mathcal{H}))^{a h}$, and therefore $e^{Z} \in \mathcal{U}_{k, d}$. Moreover, we will show that choosing a smaller neighborhood $Z$ can be written as $Z=K^{\prime}+D^{\prime}$, with $K^{\prime} \in \mathcal{K}(\mathcal{H})^{a h}, D^{\prime} \in \mathcal{D}(\mathcal{B}(\mathcal{H}))^{a h}$. In order to obtain this last assertion recall from Lemma 3.14 of [5] that there exists $\varepsilon_{0}>0$ such that if $u \in \mathcal{U}_{k, d}$ satisfies $\|u-1\|<\varepsilon_{0}$, then there exist $K^{\prime} \in \mathcal{K}(\mathcal{H})^{a h}$ and $D^{\prime} \in(\mathcal{D}(\mathcal{B}(\mathcal{H})))^{a h}$ with $u=e^{K^{\prime}+D^{\prime}}$ for $\left\|K^{\prime}\right\|,\left\|D^{\prime}\right\|,\left\|K^{\prime}+D^{\prime}\right\|<\frac{\log 2}{4}(\operatorname{see}$ Definition 2.1 and the proof of Lemma 3.14 of [5]). 
Then define the neighborhood of $b$ in $\mathcal{O}_{b}$ :

$$
\mathcal{W}_{b}=\left\{u b u^{*}: u=e^{Z} \in \mathcal{U}_{k, d}, Z \in \mathcal{B}(\mathcal{H})^{a h},\|Z\|<\log \left(1+\varepsilon_{0}\right)\right\}
$$

with $\varepsilon_{0}$ from Lemma 3.14 of [5]. Note that $\mathcal{W}_{b} \subsetneq\left(\mathcal{V}_{b} \cap \mathcal{O}_{b}\right) \subsetneq\left(\mathcal{V}_{b} \cap \mathcal{O}_{b}^{\mathcal{U}(\mathcal{H})}\right)$. It is apparent that if $u=$ $e^{Z} \in \mathcal{U}_{k, d}$, with $Z \in \mathcal{B}(\mathcal{H})^{a h},\|Z\|<\log \left(1+\varepsilon_{0}\right)$, then $\left\|e^{Z}-1\right\| \leqslant e^{\|Z\|}-1<\varepsilon_{0}$. Applying the mentioned lemma, this implies that in this case there exist $K^{\prime} \in \mathcal{K}(\mathcal{H})^{a h}$ and $D^{\prime} \in \mathcal{D}(\mathcal{B}(\mathcal{H}))^{a h}$ with $u=e^{K^{\prime}+D^{\prime}}$ for $\left\|K^{\prime}\right\|,\left\|D^{\prime}\right\|,\left\|K^{\prime}+D^{\prime}\right\|<\frac{\log 2}{4}$. Following the discussion after (5.3), if $\gamma:[0,1] \rightarrow \mathcal{O}_{b}^{\mathcal{U}(\mathcal{H})}$ is the short curve $\gamma(t)=e^{t Z} b e^{-t Z} \subset \mathcal{V}_{b}$ for $Z \in \mathcal{B}(\mathcal{H})^{a h}$ a minimal operator such that $\gamma(1)=e^{K} b e^{-K} \in \mathcal{O}_{b}$ (for $\left.K \in \mathcal{K}(\mathcal{H})^{a h}\right)$, then it must be $e^{Z}=e^{K} e^{D} \in \mathcal{U}_{k, d}$. Moreover, since $\left\|e^{Z}-1\right\|<\varepsilon_{0}$, there exist $K^{\prime} \in \mathcal{K}(\mathcal{H})^{a h}$ and $D^{\prime} \in \mathcal{D}(\mathcal{B}(\mathcal{H}))^{a h}$ satisfying

$$
e^{Z}=e^{K^{\prime}+D^{\prime}}, \text { for }\left\|K^{\prime}+D^{\prime}\right\|<\frac{\log 2}{4} \Longrightarrow Z=K^{\prime}+D^{\prime}
$$

because $Z$ and $K^{\prime}+D^{\prime}$ have norm smaller than $\pi$. Then the entire curve $\gamma:[0,1] \rightarrow \mathcal{O}_{b}^{\mathcal{U}(\mathcal{H})}$ is included in $\mathcal{O}_{b}=\mathcal{O}_{b}^{\mathcal{U}_{\mathcal{K}+\mathbb{C}}}$. In this case, being $Z=K^{\prime}+D^{\prime}$, the distance from $\rho_{0}=b$ to $\rho=e^{Z} b e^{-Z}$ is the same either if we consider the Finsler metrics in $\mathcal{O}_{b}$ or in $\mathcal{O}_{b}^{\mathcal{U}(\mathcal{H})}$ (see (3.4)). Then $\gamma(t)=e^{t Z} b e^{-t Z}=e^{t\left(K^{\prime}+D^{\prime}\right)} b e^{-t\left(K^{\prime}+D^{\prime}\right)}$, $\gamma:[0,1] \rightarrow \mathcal{O}_{b}$ defines a short curve between $b$ and $\rho=e^{K} b e^{-K}=e^{\left(K^{\prime}+D^{\prime}\right)} b e^{-\left(K^{\prime}+D^{\prime}\right)}$, with $K^{\prime}+D^{\prime}=Z$ a minimal operator of $\mathcal{K}(\mathcal{H})^{a h}+\mathcal{D}(\mathcal{B}(\mathcal{H}))^{a h}$. The statement of the theorem follows after substituting $K^{\prime}=K_{\rho}$ and $D^{\prime}=D_{\rho}$.

The element $\rho=e^{K} b e^{-K} \in \mathcal{W}_{b}$ was chosen arbitrarily, so we have proved that $\mathcal{W}_{b}$ is a geodesic neighborhood of $b$ in $\mathcal{O}_{b}$.

Remark 5.5. Observe that the unitary $e^{K_{\rho}+D_{\rho}} \in \mathcal{U}_{k, d}$ mentioned in the previous theorem might not belong to $\mathcal{K}+\mathbb{C}$, but $\gamma(t)=e^{t\left(K_{\rho}+D_{\rho}\right)} b e^{-t\left(K_{\rho}+D_{\rho}\right)} \in \mathcal{O}_{b}$ for every $t$ (see Remark 3.2).

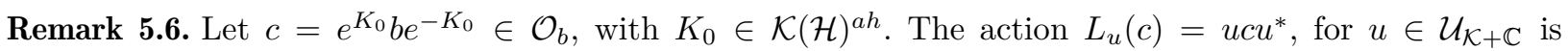
invariant for the distance defined in $\mathcal{O}_{b}$ and therefore the previous result also holds in this case. That is, there exists a geodesic neighborhood $\mathcal{W}_{c}$ of $c$ such that every $\rho \in \mathcal{W}_{c}$ is joined with $c$ by short curves included in $\mathcal{O}_{b}$ of the form $L_{e^{K_{0}}} \circ \gamma$ (for $\gamma$ the curve described in Theorem 5.4).

Here we recall some results and the notation used in [7] and state its translation to the particular example we are studying. In the work mentioned, the minimality of a curve $\gamma:\left[0, \frac{\pi}{2\|Z\|}\right] \rightarrow \mathcal{O}_{b}, \gamma(t)=e^{t Z} b e^{-t Z}$, with $Z$ be a minimal lifting of $x \in T_{b} \mathcal{O}_{b}$, was proved using a unitary reflection $r_{0}$ in a Hilbert space with certain properties (see Definition 2.4 in [7]), a representation of $\mathcal{A}$ in $B(\mathcal{H})$ with particular properties (in our case the identity representation verifies them) and a map $F: \mathcal{O}_{b} \rightarrow \operatorname{Gr}(\mathcal{H})(\operatorname{Gr}(\mathcal{H})$ is the Grassmann manifold of $\mathcal{H})$ defined by $F\left(u b u^{*}\right)=u r_{0} u^{*}$. The unitary reflection $r_{0}$ used there is

$$
r_{0}(x)=\left\{\begin{aligned}
x, & \text { if } x \in S_{b}, \\
-x, & \text { if } x \in S_{b}^{\perp},
\end{aligned}\right.
$$

where $S_{b}$ is the closure of $\Omega=\left\{x \in \mathcal{H}: x=U \xi\right.$, for $U$ a diagonal in $\left.\mathcal{U}_{\mathcal{K}+\mathbb{C}}\right\}$ and $\xi \in \mathcal{H}$ certain vector satisfying Definition 4.1 of [7].

Now, if we consider the particular case in which $Z$ is a minimal operator such that $\|Z\|=\left\|c_{j_{0}}(Z)\right\|$, $c_{j_{0}}(Z)_{j_{0}}=Z_{j_{0}, j_{0}}=0$ and $c_{j_{0}}(Z)_{j}=Z_{j, j_{0}} \neq 0$ for all $j \neq j_{0}$ (see the example of (4.2) and Lemma 6.1) we can be much more specific about $r_{0}$ and $\xi$. 
After the corresponding translation to this case

$$
\xi=i e_{j_{0}}, S_{b}=\operatorname{gen}\{\xi\} \text { and } r_{0}(x)=\left\{\begin{aligned}
x, & \text { if } x \in \operatorname{gen}\{\xi\}=\operatorname{gen}\left\{e_{j_{0}}\right\} \\
-x, & \text { if } x \in \operatorname{gen}\{\xi\}^{\perp}=\operatorname{gen}\left\{e_{j_{0}}\right\}^{\perp}
\end{aligned}\right.
$$

Moreover, $\xi$ fulfills Definition 4.1 in [7], since $Z^{2} \xi=-\|Z\|^{2} \xi, r_{0}(\xi)=\xi$ and $r_{0}(Z \xi)=-Z \xi$. Therefore, $\gamma(t)=e^{t Z} b e^{-t Z}$ minimizes length between the points $\gamma(0)=b$ and $\gamma(t)$ if $0 \leqslant t \leqslant \frac{\pi}{2\|Z\|}$.

In this context, the map $F_{\xi}: \mathcal{O}_{b} \rightarrow \mathscr{S} \subset \mathcal{H}, F_{\xi}\left(u b u^{*}\right)=u r_{0} u^{*}(\xi)$, where $\mathscr{S}$ is the unit sphere of $\mathcal{H}$, reduces length. That is, if $\delta:\left[0, t_{0}\right] \rightarrow \mathcal{O}_{b}$ and $v:\left[0, t_{0}\right] \rightarrow \mathscr{S}, v(t)=F_{\xi}(\delta(t))$ then (see Corollary 3.4 in [7])

$$
\ell(v) \leqslant L(\delta)
$$

with $L$ defined in (3.5) and $\ell$ the length in $\mathscr{S}$

The following result is an application of Theorem 4.1 and Lemma 4.2 in [7] to our context.

Proposition 5.7. Let $Z$ and $V$ be minimal operators of $(\mathcal{K}(\mathcal{H})+\mathcal{D}(\mathcal{H}))^{\text {ah }}$ and consider the following curves in $\mathcal{O}_{b}$ (defined in (3.1))

$$
\gamma(t)=e^{t Z} b e^{-t Z} \text { and } \delta(t)=e^{t V} b e^{-t V}, t>0
$$

Suppose additionally that there exists $0 \leqslant t_{0} \leqslant \min \left\{\frac{\pi}{2\left\|Z_{2}\right\|} ; \frac{\pi}{2\|V\|}\right\}$ such that $\gamma\left(t_{0}\right)=\delta\left(t_{0}\right)$.

Then, following the previous notation,

(1) $w(t)=F_{\xi}(\gamma(t))$ and $v(t)=F_{\xi}(\delta(t))$ both are geodesics in the sphere $\mathscr{S} \subset \mathcal{H}$ and

$$
\ell(w)=L(\gamma)=L(\delta)=\ell(v)
$$

(2) $\gamma$ and $\delta$ minimize length between the points $b$ and $\gamma(t)$ and $\delta(t)$ respectively, if $0 \leqslant t \leqslant t_{0}$.

(3) $v(t)=e^{t V} r_{0} e^{-t V}(\xi)=e^{t Z} r_{0} e^{-t Z}(\xi)=w(t)$, for $0 \leqslant t \leqslant t_{0}$ and $r_{0}$ the unitary reflection defined in $(5.4)$.

Proof. As it was mentioned before, items (1) and (2) are a direct consequence of Theorem 4.1 and Lemma 4.2 in [7]. If $\gamma\left(t_{0}\right)=\delta\left(t_{0}\right), w\left(t_{0}\right)$ and $v\left(t_{0}\right)$ match. Since geodesics for fixed ending points in the unit sphere $\mathscr{S}$ are unique (maximum circles), then $w(t)=v(t)$, for all $0 \leqslant t \leqslant t_{0}$.

Observe that the assumption of existence of such $t_{0}$ is possible even if $\gamma\left(t_{1}\right)=\delta\left(t_{2}\right)$ for $t_{1} \neq t_{2}$, since $\delta(t)$ can be re-scaled defining $\tilde{\delta}(t)=e^{t \frac{t_{2}}{t_{1}} V} b e^{-t \frac{t_{2}}{t_{1}} V}$, for $t \in\left[0, \frac{\pi}{2 \frac{t_{2}}{t_{1}}\|V\|}\right]$ and then $\tilde{\delta}\left(t_{1}\right)=\gamma\left(t_{1}\right)$.

Lemma 5.8. Let $b \in \mathcal{D}(\mathcal{K})^{h}$ with $b_{i, i} \neq b_{j, j}$ for $i \neq j, Z, V \in(\mathcal{K}(\mathcal{H})+\mathcal{D}(\mathcal{B}(\mathcal{H})))^{\text {ah }}$ be minimal operators such that for some $j_{0} \in \mathbb{N}\|Z\|=\left\|c_{j_{0}}(Z)\right\|, c_{j_{0}}(Z)_{j_{0}}=Z_{j_{0}, j_{0}}=0$ and $c_{j_{0}}(Z)_{j}=Z_{j, j_{0}} \neq 0$ for all $j \neq j_{0}$. Moreover, if there exists $t_{0}$ with $0<t_{0} \leqslant \frac{\pi}{2\|Z\|}$ that satisfies $e^{t_{0} Z} b e^{-t_{0} Z}=e^{s_{0} V} b e^{-s_{0} V}$, for $s_{0} \in\left[0, \frac{\pi}{2\|V\|}\right]$, then

$$
s_{0} c_{j_{0}}(V)=t_{0} c_{j_{0}}(Z)
$$

that is, the $j_{0}^{\text {th }}$ column of $Z$ is a multiple of the $j_{0}^{\text {th }}$ column of $V$. 
Proof. We can suppose that $j_{0}=1$. First, consider $W=\frac{s_{0}}{t_{0}} V$,

$$
\gamma(t)=e^{t Z} b e^{-t Z} \text { and } \delta_{0}(t)=e^{t W} b e^{-t W}
$$

for $t \in\left[0, \frac{\pi}{2\|Z\|}\right]$. Then $\gamma\left(t_{0}\right)=e^{s_{0} V} b e^{-s_{0} V}=e^{t_{0} s_{0} / t_{0} V} b e^{-t_{0} s_{0} / t_{0} V}=\delta_{0}\left(t_{0}\right)$. Moreover, since also $W$ is a minimal operator, then $\gamma$ and $\delta_{0}$ are short curves in the interval $\left[0, t_{0}\right]$ and then the length of $\left.\gamma\right|_{\left[0, t_{0}\right]}$ equals that of $\left.\delta_{0}\right|_{\left[0, t_{0}\right]}$. That is, $t_{0}\|Z\|=t_{0}\|W\|$ (see for example Theorem 4.1 of [7]) and therefore in particular

$$
\|Z\|=\|W\| .
$$

Using the preceding notations of Proposition 5.7, the assumptions made here imply that $v(t)=F_{\xi}(\gamma(t))=$ $F_{\xi}\left(\delta_{0}(t)\right)=w(t)$, for $t \in\left[0, t_{0}\right]$. Then their derivatives coincide for every $t \in\left[0, t_{0}\right]$

$$
w^{\prime}(t)=\left(W e^{t W} r_{0} e^{-t W}-e^{t W} r_{0} e^{-t W} W\right)(\xi)=\left(Z e^{t Z} r_{0} e^{-t Z}-e^{t Z} r_{0} e^{-t Z} Z\right)(\xi)=v^{\prime}(t)
$$

where $\xi=i e_{1}, \eta=\frac{c_{1}(Z)}{\left\|c_{1}(Z)\right\|}$ and the reflection $r_{0}$ are defined in (5.5) and fulfill $r_{0}(\xi)=\xi$ and $r_{0}(\eta)=-\eta$.

Then, if we evaluate (5.7) in $t=0$

$$
w^{\prime}(0)=\left(W r_{0}-r_{0} W\right)(\xi)=\left(Z r_{0}-r_{0} Z\right)(\xi)=v^{\prime}(0)
$$

Hence, since $r_{0}(\xi)=\xi$ and $r_{0}\left(c_{1}(Z)\right)=r_{0}\left(\left\|c_{1}(Z)\right\| \eta\right)=\left\|c_{1}(Z)\right\| r_{0}(\eta)=-\left\|c_{1}(Z)\right\| \eta=-c_{1}(Z)$,

$$
\begin{aligned}
W r_{0}(\xi)-r_{0} W(\xi) & =Z r_{0}(\xi)-r_{0} Z(\xi) \\
W\left(i e_{1}\right)-r_{0} W\left(i e_{1}\right) & =Z\left(i e_{1}\right)-r_{0} Z\left(i e_{1}\right) \\
i c_{1}(W)-i r_{0}\left(c_{1}(W)\right) & =i c_{1}(Z)+r_{0}\left(i c_{1}(Z)\right) \\
i\left(I-r_{0}\right)\left(c_{1}(W)\right) & =i 2 c_{1}(Z)
\end{aligned}
$$

On the other hand, if we consider the decomposition $\mathcal{H}=\operatorname{gen}\{\xi\} \oplus(\operatorname{gen}\{\xi\})^{\perp}$ then the identity operator $I$ and $r_{0}$ can be matricially described as

$$
I=\left(\begin{array}{ll}
1 & 0 \\
0 & 1
\end{array}\right) \text { and } r_{0}=\left(\begin{array}{cc}
1 & 0 \\
0 & -1
\end{array}\right)
$$

respectively. Then, (5.8) implies that $\left(I-r_{0}\right)\left(c_{1}(W)\right)=2\left(c_{1}(W)-W_{1,1} e_{1}\right)=2 c_{1}(Z)$ and then

$$
c_{1}(W)-W_{1,1} e_{1}=c_{1}(Z)
$$

and $\left\|c_{1}(Z)\right\|=\|Z\|=\|W\|$ (see (5.6)). This implies that $\left\|c_{1}(W)-W_{1,1} e_{1}\right\|=\|W\|$, and therefore

$$
W_{1,1}=0
$$

since otherwise $\left\|c_{1}(W)\right\|>\|W\|$, which is a contradiction. Therefore, returning to (5.9) we obtain that $c_{1}(W)=c_{1}(Z)$ that implies that

$$
c_{1}\left(\frac{s_{0}}{t_{0}} V\right)=c_{1}(Z)
$$

which ends the proof. 
Next, we obtain the second main result of this section.

Theorem 5.9. Let $b \in \mathcal{D}(\mathcal{K})^{h}$ with $b_{i, i} \neq b_{j, j}$ for $i \neq j, Z \in(\mathcal{K}(\mathcal{H})+\mathcal{D}(\mathcal{B}(\mathcal{H})))^{\text {ah }}$ be a minimal operator such that for some $j_{0} \in \mathbb{N}$, holds that $\|Z\|=\left\|c_{j_{0}}(Z)\right\|, c_{j_{0}}(Z)_{j_{0}}=Z_{j_{0}, j_{0}}=0, c_{j_{0}}(Z)_{j}=Z_{j, j_{0}} \neq 0$ for all $j \neq j_{0}$, the sequence $\left\{\operatorname{Diag}(Z)_{j, j}\right\}_{j \in \mathbb{N}}$ has more than one not null accumulation points, and $\gamma(t)=e^{t Z}$ be $e^{-t Z}$, for $t \in\left(0, \frac{\log 2}{8\|Z\|}\right)$.

Then there is not any minimal operator $V \in(\mathcal{K}+\mathbb{C})^{a h}$ such that $\delta(t)=e^{t V} b e^{-t V}, t \in\left[0, \frac{\pi}{2\|V\|}\right]$ satisfies $\gamma\left(t_{0}\right)=\delta\left(s_{0}\right)$ for $t_{0}, s_{0}$ in the respective domains.

Proof. As done before, we are going to prove only the case $j_{0}=1$. Suppose that there exists a minimal operator $V \in(\mathcal{K}+\mathbb{C})^{a h}$ such that $\delta\left(s_{0}\right)=e^{s_{0} V} b e^{-s_{0} V}=\gamma\left(t_{0}\right)$, for $s_{0} \in\left(0, \frac{\pi}{2\|V\|}\right]$ and $t_{0} \in\left(0, \frac{\log 2}{8\|Z\|}\right)$. Note that in particular $t_{0}<\frac{\log 2}{8\|Z\|}<\frac{\pi}{2\|Z\|}$ which implies that $\gamma$ is a short curve in all its domain.

Applying Lemma 5.3, $-\operatorname{Diag}\left(s_{0} V\right)+\operatorname{Diag}\left(t_{0} Z\right) \in \mathcal{D}(\mathcal{B}(\mathcal{H}))^{a h}$ is such that

$$
\begin{aligned}
e^{-s_{0} V} e^{t_{0} Z} & =e^{-\operatorname{Diag}\left(s_{0} V\right)+\operatorname{Diag}\left(t_{0} Z\right)} \\
\text { and }\left\|t_{0} Z\right\| & =\left\|s_{0} V\right\| .
\end{aligned}
$$

Then, the fact that $Z$ satisfies $\|Z\|=\left\|c_{1}(Z)\right\|$ (see (4.3)) implies that $V$ must fulfill that

$$
\|V\|=\frac{t_{0}}{s_{0}}\left\|c_{1}(Z)\right\|
$$

Using Lemma 5.8 follows that $t_{0} c_{1}(Z)=s_{0} c_{1}(V)$, and therefore (5.11) implies that $\|V\|=\frac{t_{0}}{s_{0}}\left\|c_{1}(Z)\right\|=$ $\frac{t_{0}}{s_{0}}\left\|\frac{s_{0}}{t_{0}} c_{1}(V)\right\|=\left\|c_{1}(V)\right\|$.

Then Lemma 6.1 implies that $c_{1}(V)$ is orthogonal to every other column of $V$. This property also holds for $c_{1}(Z)$ and the columns of $Z$. Recall the notation $\xi=i e_{1}$ and $\eta=\frac{c_{1}(Z)}{\left\|c_{1}(Z) \mid\right\|}=\frac{\frac{s_{0}}{t_{0}} c_{1}(V)}{\left\|\frac{s_{0}}{t_{0}} c_{1}(V)\right\|}=\frac{c_{1}(V)}{\left\|c_{1}(V)\right\|}$, and consider

$$
\xi+\eta=i e_{1}+\frac{c_{1}(Z)}{\left\|c_{1}(Z)\right\|}=i e_{1}+\frac{c_{1}(V)}{\left\|c_{1}(V)\right\|} \in \mathcal{H}
$$

A direct computation shows that $\xi+\eta$ is an eigenvector of $Z$ and $V$ of the eigenvalue $i\|Z\|=i\left\|c_{1}(Z)\right\|=$ $i \frac{s_{0}}{t_{0}}\left\|c_{1}(V)\right\|=i \frac{s_{0}}{t_{0}}\|V\|$ (see for example the proof of Theorem 2 in [4] for the self-adjoint case). The previous comments imply that

$$
e^{D}(\xi+\eta)=e^{-s_{0} V} e^{t_{0} Z}(\xi+\eta)=e^{-i \frac{s_{0}^{2}}{t_{0}}\|V\|} e^{i t_{0}\|Z\|}(\xi+\eta)=e^{i\left(t_{0}-s_{0}\right)\|Z\|}(\xi+\eta)
$$

where in the last equality we used $\|V\|=\frac{t_{0}}{s_{0}}\|Z\|$ and the series expansion of the exponentials. Then, using (5.10) we write

$$
e^{D}(\xi+\eta)=e^{-\operatorname{Diag}\left(s_{0} V\right)+\operatorname{Diag}\left(t_{0} Z\right)}(\xi+\eta)=e^{i\left(t_{0}-s_{0}\right)\|Z\|}(\xi+\eta) .
$$

Therefore, considering the equality in each entry of (5.12) we obtain

$$
e^{\left(-s_{0} V+t_{0} Z\right)_{j, j}}(\xi+\eta)_{j}=e^{i\left(t_{0}-s_{0}\right)\|Z\|}(\xi+\eta)_{j}
$$

for all $j \in \mathbb{N}$. The fact that $(\xi+\eta)_{j} \neq 0$ for all $j \in \mathbb{N}$ implies that $e^{\left(-s_{0} V+t_{0} Z\right)_{j, j}}=e^{i\left(t_{0}-s_{0}\right)\|Z\|}$ for every $j$. Since we suppose that $t_{0} \in\left(0, \frac{\log 2}{8\|Z\|}\right]$, then the exponent $\left(-s_{0} V+t_{0} Z\right)_{j, j}$ is small enough and we can conclude that 


$$
\left(-s_{0} V+t_{0} Z\right)_{j, j}=-s_{0} V_{j, j}+t_{0}(Z)_{j, j}=i\left(t_{0}-s_{0}\right)\|Z\|
$$

for all $j \in \mathbb{N}$. But this is a contradiction since we suppose $\operatorname{Diag}(V)=d+i \theta I$, with $d \in \mathcal{K}(\mathcal{H})^{a h}, \theta \in \mathbb{R}$, and we know that $\operatorname{Diag}(Z)$ has more than one (not null) limit. Therefore, a minimal operator $V \in(\mathcal{K}+\mathbb{C})^{a h}$ cannot form a curve $\delta(t)=e^{t V} b e^{-t V}$ that crosses $\gamma$ for $t>0$ in a certain small enough neighborhood of $b$.

Corollary 5.10. If we consider $Z_{2}$ as defined in (4.2) for every neighborhood $\mathcal{X}_{b}$ of $b$ in $\mathcal{O}_{b}$ there exist elements $\left(e^{t_{0} Z_{2}} b e^{-t_{0} Z_{2}}\right) \in \mathcal{X}_{b}$ such that there is not any short curve of the form $\delta(t)=e^{t V}$ be $e^{-t V}$ with $V \in(\mathcal{K}+\mathbb{C})^{a h}$ that joins $b$ with $e^{t_{0} Z_{2}} b e^{-t_{0} Z_{2}}$. In fact, this is true for $e^{t Z_{2}} b e^{-t Z_{2}}$, for every $t$ in certain interval.

Proof. Observe that the operator $Z_{2}$ satisfies every assumption needed by the operator $Z$ in Theorem 5.9. Then the proof is a direct application of the previous theorem.

Remark 5.11. Note that the situation mentioned in the previous corollary applies to the geodesic neighborhood $\mathcal{W}_{b}$ obtained in Theorem 5.4 even when in that case every element of $\mathcal{W}_{b}$ is reached by a short curve.

\section{Appendix}

In this section we include various results concerning minimal anti-Hermitian operators in $\mathcal{B}(\mathcal{H})^{a h}$.

Lemma 6.1. For a given fixed orthonormal basis of $\mathcal{H}$, let $V \in \mathcal{B}(\mathcal{H})^{\text {ah }}$ be such that there exists $j_{0} \in \mathbb{N}$ that satisfies $\|V\|=\left\|c_{j_{0}}(V)\right\|$ (where $c_{j}(V)$ is the $j^{\text {th }}$ column of the corresponding matrix representation of $V$ in the fixed basis). Then

$$
c_{j_{0}}(V) \perp c_{j}(V), \quad \forall j \neq j_{0} .
$$

If $\left(c_{j_{0}}(V)\right)_{j_{0}}=V_{j_{0}, j_{0}}=0$ then $V$ is a minimal operator.

Moreover, if $c_{j_{0}}(V)_{j}=V_{j, j_{0}} \neq 0$ for all $j \neq j_{0}$, then $V$ has a unique minimizing diagonal defined by

$$
V_{j, j}=-\frac{\left\langle c_{j}(V)_{\check{j}}, c_{j_{0}}(V)_{\check{j}}\right\rangle}{V_{j, j_{0}}}, \text { for } j \neq j_{0}
$$

where $c_{k}(X)_{\check{l}} \in \mathcal{H} \ominus$ gen $\left\{e_{l}\right\}$ is the element obtained after taking off the $l^{\text {th }}$ entry of $c_{k}(X) \in \mathcal{H}$.

Proof. Note that we can suppose that $j_{0}=1$ to simplify the notation. Similar considerations could be done for the $j_{0}^{\text {th }}$ column.

In the matrix representation corresponding to the fixed orthonormal basis $\left\{e_{j}\right\}_{j \in \mathbb{N}}$, we can consider

$$
x=\cos (t) e_{1}+\sin (t) e_{j}, \text { for } j \neq 1 .
$$

Observe that $\|x\|=1$, and then it must hold $\|V x\| \leqslant\|V\|$. Let us consider $f: \mathbb{R} \rightarrow \mathbb{R}$ such that

$$
\begin{aligned}
f(t) & =\left\|V\left(\cos (t) e_{1}+\sin (t) e_{j}\right)\right\|^{2}=\left\|\cos (t) V\left(e_{1}\right)+\sin (t) V\left(e_{j}\right)\right\|^{2} \\
& =\left\|\cos (t) c_{1}(V)+\sin (t) c_{j}(V)\right\|^{2} \\
& =\left\langle\cos (t) c_{1}(V)+\sin (t) c_{j}(V), \cos (t) c_{1}(V)+\sin (t) c_{j}(V)\right\rangle \\
& =\cos ^{2}(t)\left\|c_{1}(V)\right\|^{2}+\sin ^{2}(t)\left\|c_{j}(V)\right\|^{2}+2 \cos (t) \sin (t) \operatorname{Re}\left\langle c_{1}(V), c_{j}(V)\right\rangle .
\end{aligned}
$$


Then

$$
\begin{aligned}
f^{\prime}(t)= & -2 \sin (t) \cos (t)\left\|c_{1}(V)\right\|^{2}+2 \sin (t) \cos (t)\left\|c_{j}(V)\right\|^{2}+ \\
& +2\left(\cos (t)^{2}-\sin (t)^{2}\right) \operatorname{Re}\left\langle c_{1}(V), c_{j}(V)\right\rangle \\
= & \sin (2 t)\left(\left\|c_{j}\right\|^{2}-\left\|c_{1}\right\|^{2}\right)+2 \cos (2 t) \operatorname{Re}\left\langle c_{1}(V), c_{j}(V)\right\rangle .
\end{aligned}
$$

Then, if $\operatorname{Re}\left\langle c_{1}(V), c_{j}(V)\right\rangle>0$

$$
f^{\prime}(0)=2 \operatorname{Re}\left\langle c_{1}(V), c_{j}(V)\right\rangle>0 \text { and } f(0)=\left\|c_{1}(V)\right\|^{2}
$$

and then $f^{\prime}\left(t_{1}\right)>0$ for some $t_{1}>0$, which implies that $f\left(t_{1}\right)>\left\|c_{1}(V)\right\|^{2}$, a contradiction.

On the other hand, if $\operatorname{Re}\left\langle c_{1}(V), c_{j}(V)\right\rangle<0$

$$
f^{\prime}(0)=2 \operatorname{Re}\left\langle c_{1}(V), c_{j}(V)\right\rangle<0 \text { and } f(0)=\left\|c_{1}(V)\right\|^{2}
$$

and then $f^{\prime}\left(t_{2}\right)<0$ for some $t_{2}<0$, which implies that $f\left(t_{2}\right)>\left\|c_{1}(V)\right\|^{2}$, a contradiction.

Therefore it must be $\operatorname{Re}\left\langle c_{1}(V), c_{j}(V)\right\rangle=0$.

Now consider $z=\cos (t) e_{1}+i \sin (t) e_{j} \in \mathcal{H}$, that also satisfies $\|z\|=1$. Then following the steps we used in the case of $x=\cos (t) e_{1}+\sin (t) e_{j}$ but using $z$, it can be proved that $0=\operatorname{Re}(-i)\left\langle c_{1}(V), c_{j}(V)\right\rangle=$ $\operatorname{Im}\left\langle c_{1}(V), c_{j}(V)\right\rangle$

In order to prove the last part of the lemma observe that

(1) as proved in the first part of this lemma, $c_{1}(V) \perp c_{j}(V)$ for all $j \neq 1$,

(2) and the assumptions

(a) $c_{1}(V)_{1}=V_{1,1}=0$,

(b) $c_{1}(V)_{j}=V_{j, 1} \neq 0$ for $j \neq 1$

(c) and the equality $\|V\|=c_{1}(V)$

Then the proof of the minimality of $V$ follows applying Theorem 2.2 from [1] substituting $\mathcal{A}$ with $\mathcal{B}(\mathcal{H})$, $\mathcal{B}$ with $\mathcal{D}(\mathcal{B}(\mathcal{H})), \rho$ with the identity, $\xi$ with $i e_{1}$ and $Z$ with $V$. Note that we only need assumptions (1), (2)(a) and (2)(c) to prove that

$$
V^{2}\left(i e_{1}\right)=-\|V\|^{2} i e_{1} \text { and that }\left\langle V\left(i e_{1}\right), D\left(i e_{1}\right)\right\rangle=\left\langle i c_{1}(V), i D_{1,1}\right\rangle=0
$$

in order to fulfill the assumptions of Theorem 2.2 from [1].

The equality (6.2) follows after the condition $c_{1}(V) \perp c_{j}(V)$ for $j \neq 1$ and the fact that $c_{1}(V)_{j}=V_{j, 1} \neq 0$ for those $j$.

Moreover, if we consider $V+D$, for $D \neq 0$, and $D_{1,1} \neq 0$, follows that $\left\|c_{1}(V+D)\right\|=\left\|c_{1}(V)+D_{1,1} e_{1}\right\|>$ $\left\|c_{1}(V)\right\|=\|V\|$ and therefore $V+D$ cannot be minimal. Now suppose $D_{1,1}=0$. Direct computations show that

$$
\begin{aligned}
\left\|(V+D) \frac{c_{1}(V)}{\left\|c_{1}(V)\right\|}\right\| & =\frac{1}{\left\|c_{1}(V)\right\|}\left\|V c_{1}(V)+D c_{1}(V)\right\|=\frac{1}{\left\|c_{1}(V)\right\|}\|-\| c_{1}(V)\left\|^{2} e_{1}+D c_{1}(V)\right\| \\
& =\|-\| c_{1}(V)\left\|e_{1}+\frac{1}{\left\|c_{1}(V)\right\|} D c_{1}(V)\right\|>\left\|c_{1}(V)\right\|=\|V\| .
\end{aligned}
$$

In the previous strict inequality we have used (2)(a), (2)(b), $D \neq 0$ and $D_{1,1}=0$.

Then $\|V+D\|>\|V\|$ for $D \neq 0$, which implies that the diagonal defined in (6.2) is the only possible minimizing diagonal of $V$. 
Another way to prove equation (6.1) of the first part of the previous Lemma 6.1 is using Corollary 6.3 of the following theorem.

Theorem 6.2 (Sain, [9]). Let $\mathcal{H}_{1}, \mathcal{H}_{2}$ be Hilbert spaces and $T \in \mathcal{B}\left(\mathcal{H}_{1}, \mathcal{H}_{2}\right)$. Given any $x \in \mathcal{H}_{1},\|T x\|=\|T\|$ if and only if the following two conditions are satisfied:

i) $\langle x, y\rangle=0$ implies that $\langle T x, T y\rangle=0$,

ii) $\sup \{\|T y\|:\|y\|=1,\langle x, y\rangle=0\} \leqslant\|T x\|$.

Corollary 6.3. Consider $\mathcal{H}=\mathcal{H}_{1}=\mathcal{H}_{2}$ and $V \in \mathcal{B}(\mathcal{H})^{\text {ah }}$. Then there exists $j_{0} \in \mathbb{N}$ such that $\|V\|=$ $\left\|V\left(e_{j_{0}}\right)\right\|=\left\|c_{j_{0}}(V)\right\|$, if and only if

i) $\left\langle e_{j_{0}}, e_{j}\right\rangle=0$ implies that $\left\langle V e_{j_{0}}, V e_{j}\right\rangle=\left\langle c_{j_{0}}(V), c_{j}(V)\right\rangle=0$ for each $j \in \mathbb{N}, j \neq j_{0}$,

ii) $\sup \left\{\left\|c_{j}(V)\right\|: j \in \mathbb{N}\right\} \leqslant\left\|c_{j_{0}}(V)\right\|$.

Proof. The proof is a direct consequence of Theorem 6.2 after observing that in item i) of the corollary is equivalent to say that $\left\langle e_{j_{0}}, y\right\rangle=0$ implies that $\left\langle V e_{j_{0}}, V y\right\rangle=0$ for any $y \in \mathcal{H}$.

\section{References}

[1] E. Andruchow, L.E. Mata-Lorenzo, A. Mendoza, L. Recht, A. Varela, Minimal matrices and the corresponding minimal curves on flag manifolds in low dimension, Linear Algebra Appl. 430 (8-9) (2009) 1906-1928.

[2] D. Beltiţă, Smooth Homogeneous Structures in Operator Theory, Chapman \& Hall/CRC Monographs and Surveys in Pure and Applied Mathematics, vol. 137, Chapman \& Hall/CRC, Boca Raton, FL, 2006.

[3] T. Bottazzi, A. Varela, Best approximation by diagonal compact operators, Linear Algebra Appl. 439 (10) (2013) 3044-3056.

[4] T. Bottazzi, A. Varela, Minimal length curves in unitary orbits of a Hermitian compact operator, Differ. Geom. Appl. 45 (2016) 1-22.

[5] T. Bottazzi, A. Varela, Unitary subgroups and orbits of compact self-adjoint operators, Stud. Math. 238 (2) (2017) 155-176.

[6] E. Chiumiento, On normal operator logarithms, Linear Algebra Appl. 439 (2) (2013) 455-462.

[7] C.E. Durán, L.E. Mata-Lorenzo, L. Recht, Metric geometry in homogeneous spaces of the unitary group of a $C^{*}$-algebra. I. Minimal curves, Adv. Math. 184 (2) (2004) 342-366.

[8] C.E. Durán, L.E. Mata-Lorenzo, L. Recht, Metric geometry in homogeneous spaces of the unitary group of a $C^{*}$-algebra. II. Geodesics joining fixed endpoints, Integral Equ. Oper. Theory 53 (1) (2005) 33-50.

[9] D. Sain, On extreme contractions and the norm attainment set of a bounded linear operator, ArXiv, 2017.

[10] V.S. Varadarajan, Lie Groups, Lie Algebras, and Their Representations, vol. 102, Springer Science \& Business Media, 2013. 